\title{
La influencia del contexto de la interacción en la adquisición del lenguaje
}

\author{
GRACE SHUM \\ Universidad Complutense de Madrid \\ $\underbrace{}_{9}$ \\ Resumen
}

\begin{abstract}
El lenguaje no se adquiere de modo espontâneo, ni es connatural ünicamente al desarrollo biológico, sino que se adquiere y evoluciona merced a la interacción. Asi, por tanto, un favorable desarollo del lenguaje exige una condición interactiva adecuada. Para confirmar dicha tesis, se ha realizado un estudio longitudinal y transversal para observar las pautas de adquisición y desarrollo del lenguaje y niños que proceden de medios ambientales totalmente diferentes: instituciön asistencial y medio familiar. Se hizo análisis tanto cualitativo como cuantitativo en los tres niveles del lenguaje (morfosintáctico, semántico y pragmático). Los resultados mostraron diferencias evidentes entre ambos grupos de niños. Asi, los niños que pertenecen al medio institucional presentan importantes déficits en su desarrollo lingüístico.
\end{abstract}

Palabras clave: Adquisición del lenguaje, contextos de interacción, niños institucionalizados, déficits de lenguaje.

\section{The influence of contexts of interaction on language acquisition}

\begin{abstract}
Language cannot be acquired spontaneously nor can it be acquired only just because of biological evolution, but thanks to the interaction adult-child. A favourable language development needs a good interactive condition. This thesis has been tested through a longitudinal and transversal study. Child language acquisition and development pattern were observed in two groups of children of 2-3 years of age which belong to institutional and family contexts. After the quantitative and qualitative analysis a great difference was found between both groups of children, results shows that children living in institution bave a marked deficiency in languaje development.
\end{abstract}

Key words: Language acquisition, contexts of interaction, institutionalized children, language deficiency.

Dirección del autor: Universidad Complutense, Facultad de Psicología, Campus de Somosaguas, 28071 Madrid.

Original recibido: Octubre 1986. Revisión recibida: Mayo 1988. Aceptado: Junio 1988. 


\section{INTRODUCCION}

El lenguaje es el medio fundamental de comunicación y socialización, ya que la función comunicativa es su primordial propósito (Martinet, 1978; Vygotski 1973; Bruner, 1975 etc.). El niño, desde el momento de su nacimiento, por su propia supervivencia, tiene necesidad de comunicarse con su entorno. El primer paso de la interacción adulto-niño es gestual, evolucionando con la creciente importancia del lenguaje verbal. Así, por tanto, la adquisición del lenguaje infantil se desarrolla en el contexto interactivo (Snow, 1977; Bloom y Lahey, 1978; Bates, 1974; Siguán, 1978). Bruner indica que el niño aprende a expresar, a través del lenguaje verbal, una idea ya poseída que expresaba inicialmente de forma no lingüística. Así, pues, la pauta de interacción adulto-niño determina de forma importante la adquisición y evolución del lenguaje del niño. En aquellos niños que no tengan posibilidad de vivir en un medio ambiental y relacional normal o adecuado, los trastornos del lenguaje son frecuentes (Rondal, 1982; Ainsworth, 1961; Tizard, 1972; Mardomingo, 1981). Igualmente, Luria (1974) en su estudio gemelar, muestra que el factor ambiental determina el desarrollo y adquisición del lenguaje. Es decir, que aquel medio am. biente que es pobre en comunicación interactiva y personalizada incidirá de una forma negativa en la adquisición y evolución del lenguaje y, con frecuencia, puede ser la causa de trastornos en él.

El desarrollo madurativo y la organización del psiquismo en el niño, exige al adulto que presente determinados comportamientos en su relación con él (Clarke y Clarke, 1967; Bowlby, 1969; Schaffer, 1977; Schaffer y Crook, 1978; López Sánchez, 1981; Reca, 1978, Turner, 1986 etc.). Wallon señala que desde el primer momento, dicha relación es imprescindible para el desarrollo global. En un principio, el niño es un conjunto de reflejos y movimientos motores desorganizados. Esto hace que sólo pueda relacionarse con el medio a partir de las sensaciones, que son la base de la emoción. De aquí la importancia de la afectividad, que es el primer elemento de organización de la experiencia vivida. En este sentido, el adulto cumple una función no sólo de cuidador, sino de orientador, organizando al niño en función de las experiencias de éste con el único medio que tiene a su alcance, que es la afectividad (Bruner, 1975). A través de ésta, el niño se hace un ser más activo en el proceso comunicativo, transformando de ese modo su medio social. Es decir, modifica con su comportamiento las pautas de comunicación con el adulto, posibilitándole el paso a otros niveles de conocimiento y experiencia de la realidad, en la cual el adulto continúa siendo el organizador de la misma. Hacia los dos años, el lenguaje verbal comienza a convertirse en instrumento fundamental de comunicación, dejando al lenguaje analógico en un segundo plano, cumpliendo una función complementaria en el proceso comunicativo. Progresivamente, el niño va adquiriendo este instrumento que con posterioridad transformará de nuevo sus relaciones con el medio. Así, el crecimiento y la evolución psicológica del niño están en un continuo proceso dialéctico con su medio en el que el lenguaje es el mediador y organizador de dicho desarrollo (Vygotski, 1973; Luria, 1976, 1980, Rondal, 1982). Por tanto, es imposible entender la socialización si no es a través del proceso comunicativo que se establece mediante la afectividad (Wallon, 1970, Ainsworth, 1969; Schaffer, 1979; Wolff, 1967) que se expresa a través del lenguaje analógico y posteriormente, mediante el lenguaje verbal.

Por otra parte, el lenguaje es la base esencial en el desarrollo cognitivo, regu- 
lador del comportamiento y eje central para el proceso educativo (Vygotski, 1979; Luria y Yudovich, 1978, Leontiev, 1973). El agente socializador transmite al nino pautas de comportamiento social, valores normativos y conocimientos sociales a través de las palabras, que son también necesarias para expresar las necesidades, sentimientos y experiencias. Consecuentemente, en aquellos niños que viven en un medio ambiente social y comunicativo desfavorable o no adecuado, aparecerá déficit en el desarrollo lingüístico y cognitivo.

Por todo lo anterior, se propone un estudio para observar y analizar pautas de adquisición y desarrollo del lenguaje en niños que poseen problemas psicosociales y psicoafectivos, estableciendo sus diferencias con respecto a los niños que viven en un medio ambiente considerado normal o adecuado. Se intenta así averiguar los aspectos lingüísticos que se encuentran más afectados, mientras se observa, igualmente, el déficit del desarrollo cognitivo de los mismos. Para ello, proponemos un trabajo empírico que compare las consecuencias de la interacción tal como se desarrolla en una institución pública, sin problemas materiales para los niños, y en dos familias de tipo medio y ambiente normalmente apropiado, ya que en la institución las características ambientales y comunicacionales son más evidentes e incluso más objetivables que si se hubiese optado por observar el desarrollo infantil en familias con inapropiadas pautas interactivas para el desarrollo del niño.

\section{METODO}

\section{Sujetos}

El estudio consiste en observar la adquisición del lenguaje en dos grupos de niños de 2-3 años de edad y de procedencia ambiental muy diferente. Celia y Carlos viven en un medio familiar de nivel socioeconómico medio y culturalmente elevado. Ambos niños no presentan problemas afectivos ni relacionales aparentes. Mientras tanto el segundo grupo de nin̄os - Vanesa y Pablo- pertenecen a un medio institucional. Son niños que poseen factores, tanto ambientales como relacionales, muy similares, ya que tienen graves problemas familiares, económicos y afectivos, lo que les obliga a vivir en una institución de menores protegidos.

Ante todo, hay que señalar que no sólo no existe ningún tipo de carencia material en la institución donde viven Vanesa y Pablo, sino que se puede decir que los niños de dicho centro disfrutan todas sus clases de bienes que precisan para su desarrollo y la satisfacción de sus necesidades. El nivel de vida de estos niños sobrepasa al de muchos niños de familia acomodada. Sin embargo, hay algunas características en el contexto institucional que marcan diferencias sustanciales con el marco familiar, que definen la interacción adulto-niño, y que inciden en la adquisición del lenguaje. Características que ya han sido señaladas en un estudio experimental realizado por Mardomingo (1981) en esta institución, y que pueden definirse en los siguientes puntos:

1. Respecto a la estimulación - sensorial perceptiva - proporcionada por el cuidador es no sólo escasa y poco variada sino también de calidad deficiente.

2. El adulto no proporciona al niño las oportunidades suficientes para ejercitar su motricidad.

3. La calidad de las respuestas del cuidador a las demandas del niño está di- 
ficultada por el número de niños que el cuidador tiene a su cargo.

4. La existencia de varios cuidadores del niño y el cambio continuo de los mismos (3 turnos diarios).

5. La escasa calidad del intercambio emocional entre el niño y el cuidador (sontisas, caricias, expresiones verbales cariñosas, etc.).

Estas variables, según la autora, están relacionadas con una serie de trastornos a nivel de desarrollo (edad ósea, peso, talla) y en otros aspectos como la conducta personal, social y adaptiva, apareciendo además cuadros de trastornos del tipo de polifagia, anorexia, infecciones, trastornos digestivos, etc. Por otra parte, esto produce un deterioro en los aspectos expresivo y comunicativo que incide tanto en el vocabulario, la estructura del lenguaje como en la capacidad de razonamiento abstracto y concreto.

\section{Procedimiento}

Se utilizó un método transversal y longitudinal, sirviéndose de un video portátil para la recogida de datos. Las observaciones se realizaron en un contexto ambiental totalmente natural y habitual en el niño. La duración de las observaciones fue de 30 minutos con un intervalo de 30 días aproximadamente.

El primer lugar, se estudió la evolución global del lenguaje de cada uno de los niños a través de la evaluación de la longitud media de los enunciados (LME). Con el fin de observar la calidad del ajuste de dicho desarrollo, se obtuvo la recta de regresión para dicha media. En un intento de completar el análisis, se calculó la desviación típica para medir, de alguna forma, la extensión del número de morfemas por enunciado que eran capaces de producir los niños; a través de lo cual se refleja la capacidad sintáctica y semántica del niño. Para hacer comparables dichas medidas, se calculó el coeficiente de variación de la desviación típica respecto a la media.

Posteriormente se analizaron los mensajes de los niños en tres niveles lingüísticos: morfosintáctico, semántico y pragmático. El análisis morfosintáctico se realizó en base al sistema de las categorías y subcategorías gramaticales morfológicas de la lengua castellana, teniendo en cuenta su momento de aparición y el orden en que se manifiestó su adquisición en el lenguaje expresivo. Se observó también el proceso de aprendizaje en cuanto a la concordancia de género y número. Para comparar las categorías entre sí y con respecto a cada niño, se cuantificaron las frecuencias por categorías, transformando los datos en porcentajes. Además, en el estudio transversal, se utilizaron las puntuaciones medias de cada grupo de niños para establecer ciertas comparaciones. También se procedió a un tratamiento estadístico de las frecuencias absolutas de las categorías gramaticales, hallando los coeficientes de correlación entre ellas y analizando aquellas puntuaciones estadísticamente significativas, buscando las posibles causas de dicha significación.

En cuanto al análisis semántico, se examinó la evolución lexical del niño, ya que a través de ésta no sólo se observa el conocimiento del mundo físico y social, sino también refleja la capacidad lingüística del niño, en el sentido que cuanto mayor sea el caudal léxico más rico será su campo semántico. Para ello, se contabilizó el número de palabras semánticamente diferentes que iban apareciendo durante todo el período de observación. A través de dicha medida, se puede obtener el caudal léxico que posee cada niño. También se cuantificó 
el incremento de las palabras nuevas por categorías morfológicas, para precisar la evolución de la riqueza semántica. Además, se establecieron comparaciones entre los niños con respecto al momento de aparición de cada categoría y se consideró el número de palabras distintas utilizadas por el niño en cada observación. Por último, se analizó la aparición de subextensión y sobreextensión en el significado de los mensajes empleados.

Para el estudio pragmático se estableció un sistema de categorías que designan las funciones que los enunciados desempeñan en el proceso de interacción. Se precisa un modelo que, en cierta forma, sea capaz de recoger las distintas funciones del lenguaje que utilizan los niños en este período de desarrollo. Dichas categorías son exhaustivas y excluyentes entre sí, de tal modo que no se solapan unas a otras y pueden observarse claramente las diferencias en el uso del lenguaje de los niños. Fundamentalmente se utilizaron dos funciones principales —enunciativa e imperativa -, según son recogidas por Dale (1980b). Al mismo tiempo, se clasificaron dichas funciones en siete subcategorías: referencia a objeto o acción presente; referencia a objeto ausente, acción futura o pasa-

Figura 1

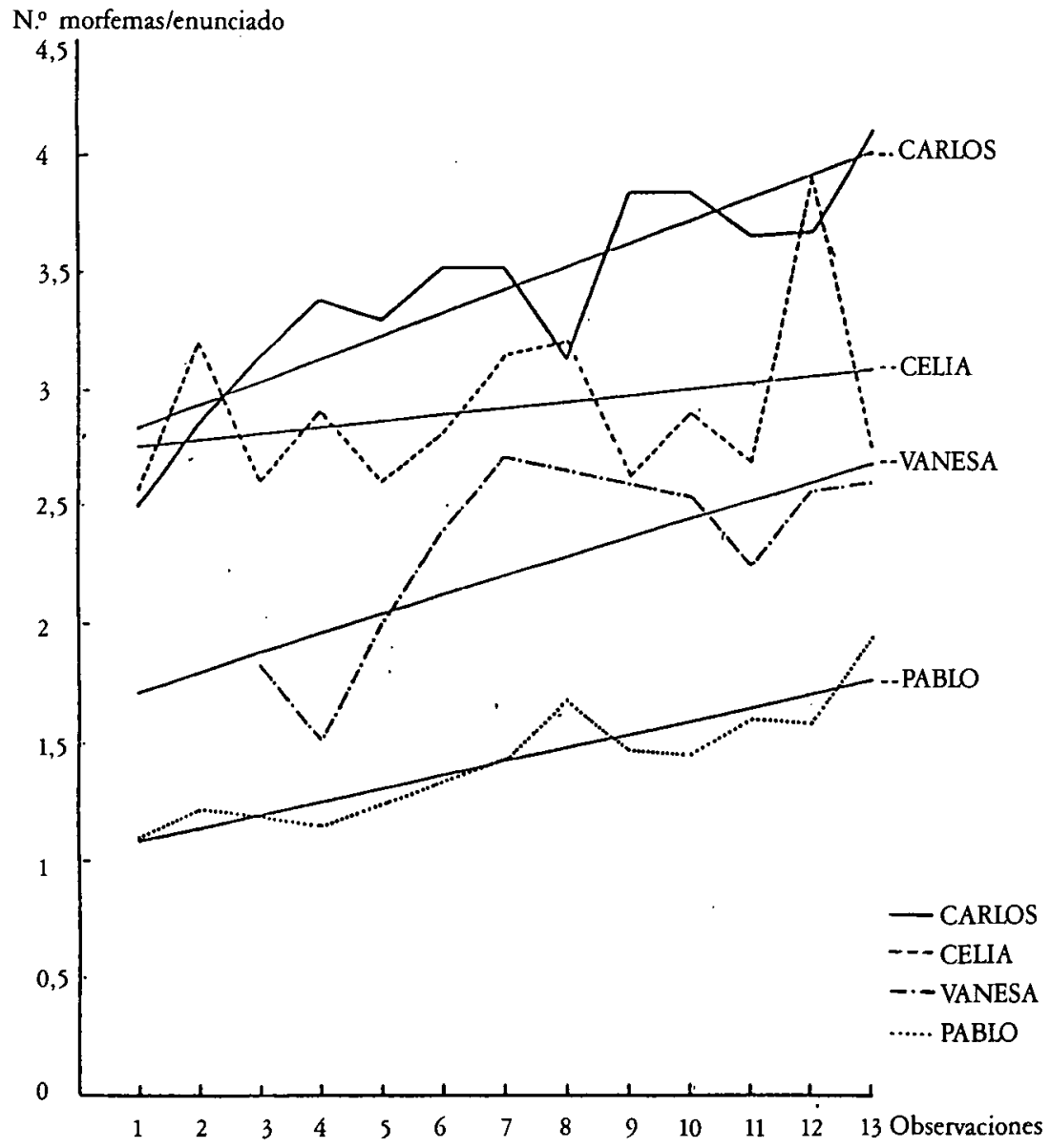


da; enunciado sin referencia; demanda de objeto o acción presente; demanda de objeto ausente, acción futura o pasada; demanda de información con interrogación abierta o cerrada; demanda de atención. Además, se incorporaron aspectos del modelo de Miller (1981): imitación, imitación exclusiva y repetición; ya que a través de dichas categorías podemos detectar la actitud comunicativa del niño. Son categorías que cumplen fundamentalmente la función fática de Jakobson. Finalmente se analizaron todos aquellos mensajes que son incorrectos pragmáticamente. Mediante dicho análisis se puede detectar mejor la capacidad del lenguaje tanto comprensiva como expresiva, que no se registra analizando sólo la corrección morfosintáctica o semántica de los enunciados.

\section{ANALISIS DE LOS RESULTADOS}

\section{Análisis global del desarrollo del lenguaje}

Según los datos analizados, en una primera observación resulta evidente que la evolución del lenguaje de los niños es ascendente. Sin embargo, existen marcadas diferencias, ya que el ritmo del avance difiere de unos a otros. Según la recta de regresión de la LME (figura 1), el mayor avance se da en Carlos, con gran diferencia sobre las demás, mientras que el menor avance lo tenía Celia. En cualquier caso, la puntuación de los niños que viven con sus padres es superior a los del medio institucional. En cierto momento, las curvas de LME de Vanesa y Celia obtienen una puntuación semejante, pero hay que tener en cuenta que los puntos en los que se aproximan coinciden con las puntuaciones más bajos de Celia y más altas de Vanesa. Además, se observa que Celia y Carlos producen enunciados con un número de morfemas superior a los que emiten los niños institucionalizados (Vanesa y Pablo) (Tabla I). Igualmente, en el aná-

TABLA I

Nümero máximo de morfemas por enunciado alcanzado en cada observación

\begin{tabular}{|c|c|c|c|c|}
\hline $\begin{array}{c}\text { Orden de } \\
\text { observación }\end{array}$ & Celia & Carlos & Vanesa & Pablo \\
\hline $1 .^{2}$ & 12 & 8 & - & 2 \\
$2 .^{2}$ & 9 & 7 & - & 2 \\
$3 .^{2}$ & 11 & 18 & 5 & 3 \\
$4 .^{2}$ & 12 & 18 & 5 & 2 \\
$5 .^{2}$ & 10 & 12 & 6 & - \\
$6 .^{2}$ & 9 & 14 & 9 & - \\
$7 .^{2}$ & 10 & 10 & 8 & 4 \\
$8 .^{2}$ & 13 & 12 & - & 6 \\
$9 .^{2}$ & 10 & 13 & - & 5 \\
$10 .^{2}$ & 14 & 16 & 8 & 4 \\
$11 .^{2}$ & 10 & 15 & 8 & 5 \\
$12 .^{2}$ & 13 & 14 & 10 & 5 \\
$13 .^{2}$ & 11 & 17 & 9 & 5 \\
\hline
\end{tabular}


lisis estadístico de las desviaciones típicas y coeficientes de variación de la longitud de los enunciados, también aparecen direrencias evidentes (Tabla II), lo cual indica claramente que la estructura sintáctica del lenguaje de Celia y Carlos es mucho más compleja y más rica.

\section{TABLA II}

Desviación típica y coeficiente de variación relativa respecto a la longitud media de los enunciados

\begin{tabular}{|c|c|c|c|c|c|c|c|c|}
\hline \multirow{2}{*}{$\begin{array}{c}\text { Orden de } \\
\text { observación }\end{array}$} & \multicolumn{2}{|c|}{ Celia } & \multicolumn{2}{c|}{ Carlos } & \multicolumn{2}{c|}{ Vanesa } & \multicolumn{2}{c|}{ Pablo } \\
\cline { 2 - 8 } & $\sigma$ & C.V. & $\sigma$ & C.V. & $\sigma$ & C.V. & $\sigma$ & C.V. \\
\hline 1 & 1,79 & 0,69 & 1,81 & 0,72 & - & - & 0,30 & 0,28 \\
2 & 1,89 & 0,59 & 1,62 & 0,57 & - & - & 0,41 & 0,34 \\
3 & 1,80 & 0,56 & 2,16 & 0,69 & 1,03 & 0,56 & 0,44 & 0,38 \\
4 & 2,10 & 0,72 & 2,45 & 0,72 & 0,88 & 0,58 & 0,34 & 0,30 \\
5 & 2,01 & 0,76 & 2,42 & 0,74 & 1,15 & 0,58 & - & - \\
6 & 1,97 & 0,70 & 2,74 & 0,78 & 1,63 & 0,69 & - & - \\
7 & 1,88 & 0,60 & 2,22 & 0,63 & 1,66 & 0,63 & 0,66 & 0,46 \\
8 & 2,26 & 0,70 & 2,36 & 0,75 & - & - & 0,91 & 0,54 \\
9 & 1,64 & 0,63 & 2,58 & 0,67 & - & - & 0,84 & 0,57 \\
10 & 2,25 & 0,78 & 2,95 & 0,77 & 1,70 & 0,67 & 0,69 & 0,48 \\
11 & 1,91 & 0,71 & 2,82 & 0,77 & 1,67 & 0,74 & 0,85 & 0,53 \\
12 & 2,55 & 0,66 & 2,77 & 0,76 & 1,82 & 0,71 & 0,93 & 0,59 \\
13 & 1,95 & 0,71 & 3,05 & 0,74 & 1,53 & 0,59 & 1,16 & 0,60 \\
\hline
\end{tabular}

Comparando el ritmo de adquisición del lenguaje de Carlos y Celia con el sujeto de estudio de Hernández Pina (1984), se observa que el momento de adquisición de las categorías morfológicas de estos niños coincide prácticamente. Así, se puede considerar que ambos niños presentan un desarrollo del lenguaje adecuado a la edad cronológica. Carlos presenta globalmente una evolución más regular, mientras que Celia manifiesta un mejor desarrollo en las primeras observaciones, aunque a lo largo del período de estudio registra fluctuaciones a todos los niveles.

En cuanto a Vanesa y Pablo, presentan un retraso notable en todos los niveles. La adquisición de Pablo es muy tardía, a los dos años se encuentra en la etapa holofrástica y no ha superado el período telegráfico (enunciados de dos a tres palabras) al final del estudio. Vanesa, aunque su nivel de evolución lingüística es superior a Pablo, presenta un desarrollo poco homogéneo, destacando especialmente su desfase en el nivel pragmático (Figura 4).

Análisis morfosintáctico de la evolución lingüistica de los niños

El momento de aparición de las categorías gramaticales es más tardío en los niños institucionalizados. Aparte de ser muy pobre e insuficiente el nivel de uso de las mismas, algunas de ellas apenas aparecen en todo el período de ob- 


\section{4}

Figura 2

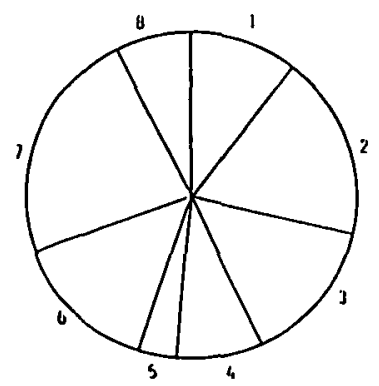

CELIA

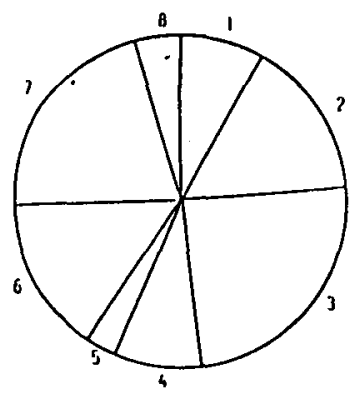

VANESA

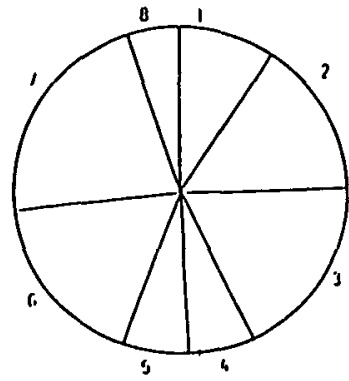

CARLOS

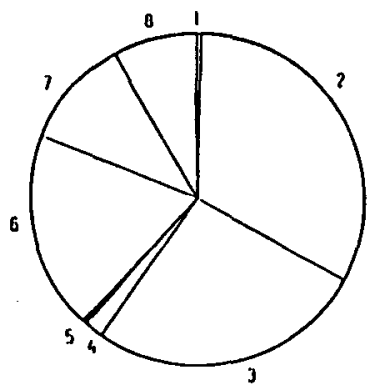

PABLO

1. Artículo; 2. Sustantivo; 3. Adverbio; 4. Preposición; 5. Conjunción; 6. Pronombre;

7. Verbo; 8. Adjetivo

Proporción de uso de las categorias morfológicas (contabilizando errores).

servación (Figura 2) y otras son escasas. Así ocurre con el uso de los artículos plurales determinados y, en cuanto al artículo plural indeterminado, no se observa su utilización tanto en Vanesa como en Pablo durante todo el período de observación. El sustantivo plural, la preposición y las subcategorías temporales del adverbio son prácticamente inexistentes en los niños anteriormente mencionados.

Se puede decir que Pablo, en sus primeras observaciones, se encuentra todavía en la etapa holofrástica. Sus mensajes a los dos años eran del tipo de:

- aallo (caballo)

- cheche (coche)

- tae (trae)

Los sustantivos que usa Pablo en este período cumplen la función morfosintáctica de «objeto», son utilizados para señalar o designar aquellas cosas que aparecen en el contexto y que el niño conoce. La función asujeto» no aparece hasta más tarde y comienza siendo el pronombre đyo» el que la desempeña.

Hasta los 2;2 años, no comienza a construir Pablo enunciados de dos palabras, etapa en la que continúa hasta los 2;11 años. Durante dicho período los mensajes del niño son del tipo de:

- más aallo (más caballo) 
- saca mamá

- melo etá (caramelo está)

Finalmente, 3 años, produce enunciados de tres y cuatro palabras:

- papá tá aquí (papá está aquí)

- no abe eto (no abre esto)

- oto coco a efante (otro ojo de elefante)

Vanesa, a los 2; 2 años, utiliza enunciados de dos y tres palabras, de tipo de:

- patito bebe

- no tene piena (no tiene pierna)

- a domir (a dormir)

- a patito sí

Entre los 2;3 años y los 2;5, sólo alcanza a emitir enunciados de tres y cuatro palabras, como por ejemplo:

- él no come

- porque tene pupa (porque tiene pupa)

- este es el yayo

- dame el melo.(dame el caramelo)

- metele a éste

A partir de entonces y hasta los 3 años construye fundamentelmente enunciados de cinco palabras y esporádicamente produce mensajes de seis palabras. Por último, en este período aparecieron algunos enunciados de siete palabras. El tipo de enunciados de la niña entonces es:

- en esta camión no cabe

- que me ha dado un cacharro

- ¿a dónde está la pueta esta?

- ¿a donde está el avión que monta?

Entre Celia y Carlos no se dan diferencias sustanciales aparentes; desde los dos años, sus enunciados tienen ya una extensión de hasta siete palabras, aunque en estas últimas aparecían frecuentemente errores morfosintácticos. Hasta los 2;3 años los enunciados de ambos niños son del tipo:

Celia

- doy un salto?

- miralque trenecillo

- aquí hay una barquita

- yo como chupa-chups con azúcar

- este aparato es de un astronautita?

- el Támesis está por donde Londres
Carlos

- me gusta el pan

- se ha sentado en el orinal

- vamos a hacer una cosa

- aquí también cabe otra gradote

- los patos del parque hace así

- es que me ba manchado de tierra

En una segunda etapa, de 2;3 a 2;6 años, ambos niños emiten sin problemas enunciados de hasta nueve palabras:

Celia

- quiere decir que el chupa-chups tie-

ne chicles

- ponle un coche a ver si anda

- no puedes meter este libro/mamálen

esta bolsa?
Carlos

- ese no es el coche de bomberos

- que hay un poco pegado a la puerta

- porque me da igual hacer esto que esto 


\section{6}

Sobre los 3;0 años, aunque no muy a menudo, formulan mensajes más completos, incluso oraciones subordinadas:

Celia

- este tiene unas cosas/unos bichos

que son largos

- mañana me vas a traer un toro que

es negro

- se llama acacia porque tiene dos árboles que son acacias
Carlos

- cojo la carretilla y me la llevo a la calle

- en la feria que habia un generally asi me disparaba

- y hay un coche dentro y me coge y me asusta y me da un cachetón

Así, a través del estudio longitudinal, es evidente que la estructura sintáctica y el contenido de los enunciados de los niños de medio familiar varían sustancialmente respecto a los de medio institucional. Celia y Carlos no sólo elaboran enunciados simples correctamente, sino que ya son capaces de formular mensajes complejos. Vanesa utiliza la conjunción «y» sólo como nexo entre palabras. Sin embargo, Carlos y Celia la utilizan, además, como nexo entre oraciones. Esto muestra su mayor capacidad sintáctica, ya que son capaces de coordinar oraciones entre sí. Además, utilizan también con este mismo fin conjunciones como «si», "pero», «que»y «porque».

\section{Análisis semántico del lenguaje}

Lo mismo ocurre con el análisis semántico, ya que Vanesa y Pablo presentan diferencias sustanciales con respecto a Carlos y Celia. Ambos niños del medio familiar adquieren mayor número de palabras que los de medio institucional (Figura 3). Esto se manifiesta en menor número de palabras emitidas por observación, en el total de palabras distintas y en el escaso incremento de nuevos vocablos. Además, los niños de medio familiar emiten enunciados más elaborados y complejos, y la puntuación de la LME es mayor que la de los niños institucionalizados; muestran, consecuentemente, un superior nivel semántico.

Con respecto al problema de extensión de los significados, se observa que la subextensión aparece en los cuatro niños. Clark (1976) explica este hecho relacionándolo con el desarrollo madurativo y el nivel de comprensión alcanzado. No obstante, la diferencia radica en que Celia y Carlos conocen un número superior de características y funciones de los referentes, lo cual manifiesta su mejor desarrollo comprensivo, frente a los niños de medio institucional. Hay muchos referentes que son desconocidos para Vanesa y Pablo, y estos niños sustituyen su denominación por pronombres demostrativos y adverbios de lugar (aquí, allí y ahí), que cumplen la función deíctica. Según indica Bruner (1983b), a medida que el niño consolida el lenguaje disminuyen estas funciones. Por esta misma razón, se fuede decir que el lenguaje de Vanesa y Pablo está poco consolidado.

El problema de sobreextensión en Carlos y Celia desaparece muy tempranamente, mientras que Vanesa y Pablo lo presentan a lo largo de todo el período de observación. Este aspecto merece un tratamiento más extenso, al que no da lugar este artículo. 
FIGURA 3

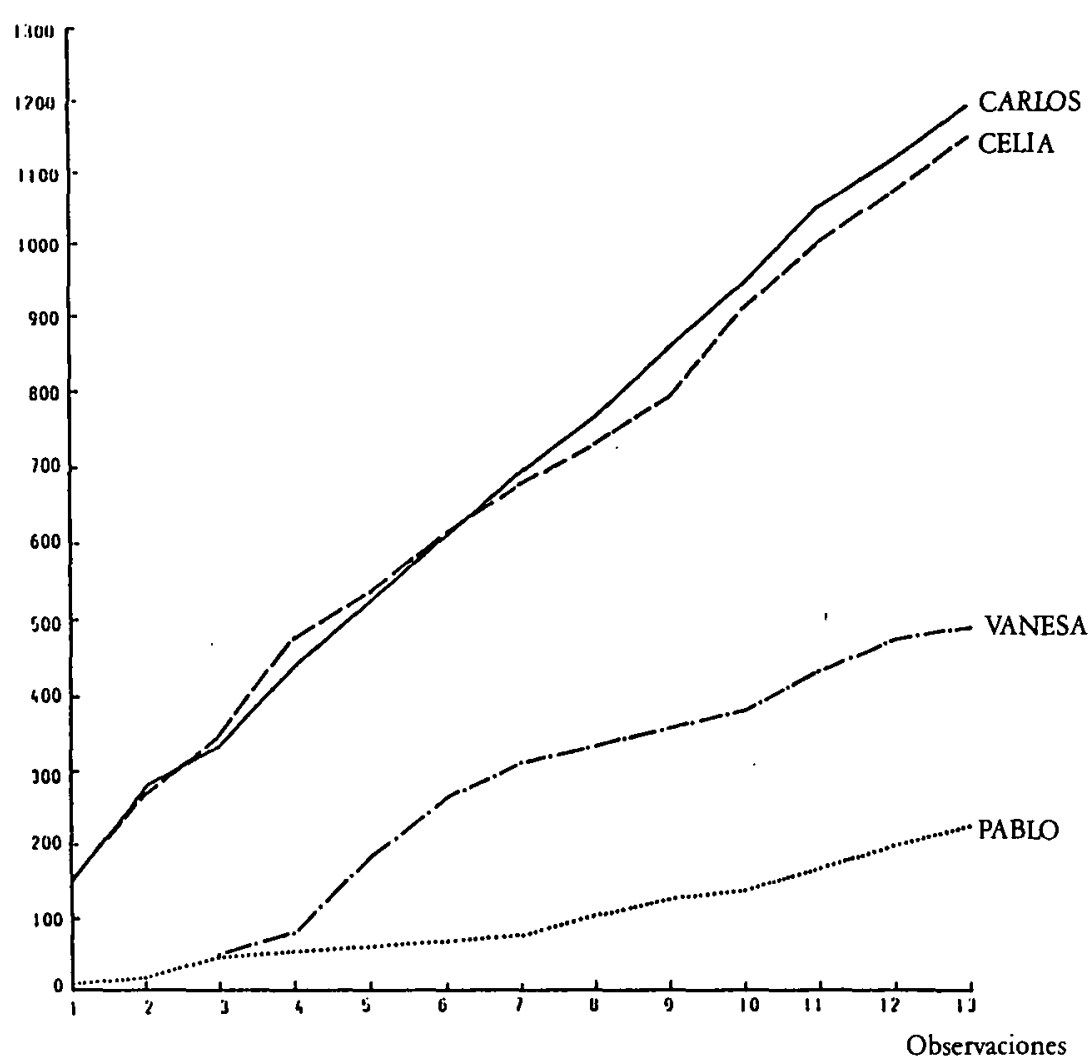

Adquisición del léxico. Palabras nuevas por observación (frecuencias acumuladas).

Para ilustrar lo anteriormente expuesto, se presenta una pequeña muestra de los mensajes tipos de Celia, Carlos y Vanesa. No se incluyen los enunciados de Pablo, dado que su desarrollo lingüístico es todavía inferior al de Vanesa, como ya se ha indicado.

Carlos

- "ya apareció el coche"

- eme asustón

- «ub!lel coche aplastó el - ase cae el coches conductor

- "preparado/unoldos/ _ "ya estálya estā" pum!

- "ime desnudas?"
Vanesa

- "ya está el avión*

- "quita eso"

- equítame esto
Celia

- aesque tenía un poco de miedo

*voy a prepararle un café sin cafeinado:

- voy a desnudar a mi Tosky" 


\section{8}

\begin{tabular}{|c|c|c|}
\hline$-\cdots-\cdots-\cdots$ & - "esto me hace pupa" & $\begin{array}{l}\text { - } \text { pequeña pellizca la } \\
\text { pequeña }\end{array}$ \\
\hline $\begin{array}{l}\text { - "me escondo" } \\
\text { - "dicen los niños que }\end{array}$ & - "no está" & - aescóndeme \\
\hline quieren sugus y ositos» & - "este quiere queso" & $\begin{array}{l}\text { - papáldice que te llamas } \\
\text { José» }\end{array}$ \\
\hline _- "mami/me ayudas?" & - "abora tü» & $\begin{array}{l}\text { - "te voy a ayudar a bacer } \\
\text { esto" }\end{array}$ \\
\hline - "nolno me molesta" & $\begin{array}{l}\text { - "abora me voy/ya no } \\
\text { vengo más» }\end{array}$ & - "me enfado" \\
\hline — "se destroza el aviōn» & - "se rompe el coche" & - "me estropeas el triciclo" \\
\hline "este sirve para comen & - aeste es para comen & - "te sirvo un café?» \\
\hline -ーーーーーー & - "son iguales" & - "voy a medir la ventana" \\
\hline $\begin{array}{l}\text { - "dice que sugus" } \\
\text { - "me ba tragado al }\end{array}$ & - "dame un caramelo" & - "no necesito pañales" \\
\hline $\begin{array}{l}\text { masticarlo" } \\
- \text { este funciona asi» }\end{array}$ & $\begin{array}{l}\text { - "ya lo comi» } \\
\text { - "este andaleste no } \\
\text { anda }\end{array}$ & $-\cdots-\cdots-\cdots$ \\
\hline
\end{tabular}

En los ejemplos citados, se puede observar los diferentes modos para expresar contenidos similares que realizan estos niños, mediante los cuales se pone de manifiesto que los enunciados de Vanesa son conceptualmente más generales y menos ricos.

El uso de adjetivos refuerza la hipótesis de la influencia del medio en la adquisición del lenguaje. La diferencia de aparición de las distintas clases de adjetivos, y la escasa variedad de ellos dentro de cada categoría, incapacitan a los niños de medio institucional para establecer y organizar las distintas experiencias en función de los atributos que las definen. Por supuesto, esto no quiere decir que los niños de medio familiar tengan un conocimiento real de todas las caractérísticas de los objetos, su edad madurativa no lo posibilita todavía. Sin embargo, son capaces de diferenciar colores, cantidad o forma de las cosas, no por el conocimiento de los conceptos, sino porque los conocen en función de la asociación y disociación mediante sus experiencias vividas, según afirma Alarcos (1970).

Los mensajes de Vanesa, aunque son un poco más elaborados que los de $\mathrm{Pa}$ blo, no dejan de ser rígidos, repetitivos y pobres en variedad de categorías morfológicas y semánticas.

\section{Análisis pragmático del lenguaje de los niños}

En línea general, existe una gran diferencia en el uso de las cuatro grandes categorías pragmáticas en los cuatro niños. La función enunciativa es la categoría más usada. La otra función que también emplean los niños con mucha frecuencia es la imperativa, aunque las diferencias que aparecen entre ambos grupos de niños son, en este caso, más grandes (Figura 4). De nuevo, Pablo supone una excepción, puesto que presenta mayor número de mensajes encuadrados en la categoría de imitación que en la imperativa. Esto es clara consecuencia del nivel de desarrollo del lenguaje verbal en el que se encuentra el niño. La función imitativa es el medio fundamental en Pablo para mantener la comunicación con el adulto y también para su aprendizaje.

Las diferencias pragmáticas que se presentan entre ambos grupos de niños también reflejan el inferior desarrollo del lenguaje de los de medio institucio- 


\section{9}

FIgURA 4

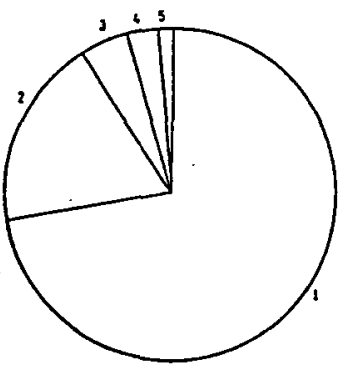

CELIA

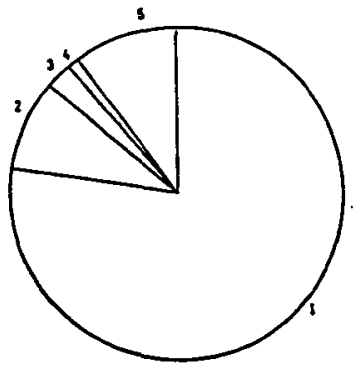

VANESA

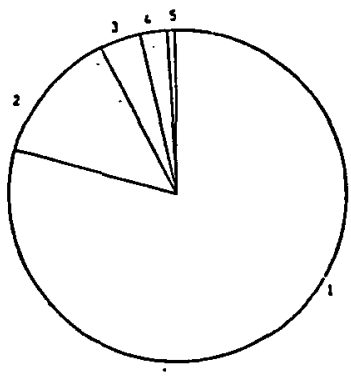

CARLOS

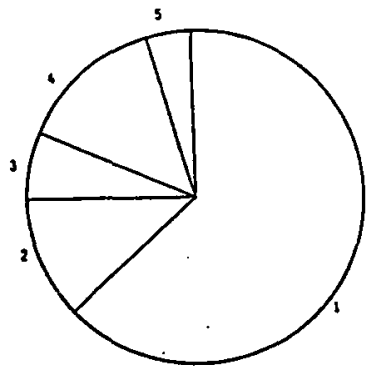

PABLO

1. Enunciativos; 2. Imperativos; 3. Repetición; 4. Imitación; 5. Inadecuación

Utilización pragmática del lenguaje. Proporción de uso de las categorías (media de todas las observaciones).

nal. Vanesa y Pablo apenas denominan y mucho menos demandan los objetos o acciones ausentes. Además, a través de la demanda de información, observamos un mayor nivel de complejización sintáctica en los niños de medio familiar, ya que son capaces de formular sus preguntas con categorías gramaticales específicas, mientras que Vanesa y Pablo todavía continúan utilizando elementos paralingüísticos para diferenciar las funciones enunciativas e interrogativas.

Un dato más que muestra el menor desarrollo lingüístico y cognitivo de los niños de la institución, y que está estrechamente vinculado al comportamiento social, es la estructura sintáctica al demandar objetos y acciones. Vanesa y Pablo utilizan la forma más elemental de expresión, es decir, con el verbo en forma imperativa, mientras que Celia y Carlos se expresan de forma más elaborada y adaptada al comportamiento social exigido.

Los niños institucionalizados presentan un elevado porcentaje de mensajes pragmáticamente inadecuados. En el caso de Pablo se puede considerar por su bajo nivel de desarrollo lingüístico. Mientras que en Vanesa aparece un claro desfase lingüístico que no se manifiesta tan evidente en el aspecto sintáctico o semántico, lo que indica que el desarrollo de la niña no es el que aparenta. Las estructuras sintácticas que usa para expresarse pueden ser las oídas en un contexto determinado, sin embargo, no es capaz de generalizarlas a otros contextos de forma adecuada. 


\section{DISCUSION Y CONCLUSIONES}

Según los datos analizados, es evidente que existe un déficit importante en la adquisición y evolución del lenguaje en los niños de medio institucional. Observamos que hay diferencia en el momento de aparición de las categorías morfológicas, así como en la variedad del uso dentro de cada categoría, entre ambos grupos de niños. En cuanto al nivel morfosintáctico, existe clara diferencia, como muestra la LME, que también se manifiesta con respecto a la complejidad sintáctica.

A su vez, en el campo semántico se observa una gran diferencia, tanto en cantidad como en cualidad. Es decir, que no sólo hay diferencia en el caudal léxico, sino también en la calidad de la expresión de los enunciados (variedad en contenidos, precisión en información etc.).

Pragmáticamente, no sólo aparece diferencia en la frecuencia del uso de las categorías pragmáticas establecidas, sino también en la forma de la expresión lingüistica, que exige mejor conocimiento lingüístico y social.

El análisis global del lenguaje, sobre todo el aspecto pragmático del mismo, es un buen indicador para observar el nivel de desarrollo cognitivo infantil ( $\mathrm{Da}$ le, 1980). Si tenemos en cuenta que los sujetos estudiados (niños de 2 a 3 años) se encuentran en la edad en que da comienzo la aparición de la función simbólica, y la interiorización de los esquemas de acción en representaciones (Piaget, 1973), su nivel de conocimiento es muy limitado y las experiencias vividas todavía escasas. Por lo tanto, por medio de un estudio longitudinal (que incluye análisis cualitativos y cualitativos), a través de la observación natural es posible recoger una muestra representativa de situaciones que permite evaluar el nivel de desarrollo alcanzado y la evolución lingüística y cognitiva de los mismos.

Analizando la forma de construcción, el contenido, y el uso de los enunciados de los niños se puede detectar de manera bastante aproximada las diferencias en el nivel y grado de conocimiento alcanzado por los sujetos. Esto se evidencia en el menor número de referentes utilizados por los niños de la institución, así como, en la escasa denominación que hacen de los atributos y cualidades de los objetos y acciones, reflejo de su bajo nivel de conocimiento del mundo físico y social. Esto se pone de relieve de manera más clara en el análisis pormenorizado de las categorías morfológicas, así como en la expresión temporal de las acciones y la referencia a la presencia o ausencia de los objetos, que no ha lugar en este artículo, ya que exigiría una exposición muy extensa, aspectos en los cuales los niños de la institución alcanzan niveles muy bajos.

Teniendo en cuenta todos los datos anteriormente señalados, y dado que los niños de la institución no presentan problemas orgánicos, se puede afirmar que las diferencias encontradas en la adquisición y evolución del lenguaje se deben fundamentalmente al medio ambiente. Así, pues, el lenguaje no se desarrolla en el niño de forma tan natural ni tan espontánea, sino que exige ciertas condiciones en su contexto social para que éste se dé de un modo favorable. Esto es:

- interacción diádica y personalizada.

- transformación de la situación de la interacción según la exigencia de desarrollo madurativo intelectual del niño.

- expresión afectiva del adulto en la comunicación adulto-niño. Condiciones que no se cumplen siempre en el contexto donde se desarrolla el niño y que se refleja de un modo muy explícito en el ambiente institucional, al que pertenece uno de los grupos de sujetos estudiados. Estos niños tienen sus nece- 
sidades cubiertas antes de tener que manifestarlas en función de un horario previamente establecido. Las relaciones que establecen con sus cuidadores se realizan de un modo mecánico, sin acompañamiento del lenguaje - verbal o gestual - afectivo, que normalmente las madres utilizan con sus hijos. De este modo les estimulan a la comunicación, necesaria para la adquisición del lenguaje (Siguán, 1978). Además, la monotonía de su vida cotidiana no les proporciona la estimulación suficiente para dar significación a las experiencias vividas. Así, por tanto, parece haber una relación bastante evidente entre la privación de las condiciones anteriormente mencionadas y el déficit en la adquisición del lenguaje infantil.

\section{Referencias}

AINSWORTH, M. (1969). Object relations, dependency and attachment: a theorical review of infantmother relationship. Child Development.

AINSWORTH, M., y BEL, S. (1974). Mother-infant interaction and the development of competence. En K. Connolly y J. Bruner (eds.), The growth of competence, Nueva York: Academic Press.

ALARCOS, LL., E. (1976). La adquisición del lenguaje por el niño. En VV.AA.: La adquisición del lenguaje por el niño. Desórdenes, funciones secundarias y representaciones gräfícas del lenguaje, 9-42. Buenos Aires: Nueva Visión.

BATES, E. (1974). Acquisition of pragmatic competence. J. Child Lang. I, 277-281. (1976). Language and context: the acquisition of pragmatics. Nueva York: Academic Press.

Вцоом, L. (1970). Semantic features in language acquisition. En Conference on research in the lenguage of the mentally retarded, University of Kansas.

BLOM, L, y LAHEY, M. (1978). Language development and Language disorders. Nueva York: John Wiley \& Sons.

BRONCKART, J. P. (1981). Procesos y estructuras del desarrollo del lenguaje. Infancia y Aprendizaje, Monografia $n .^{\circ} 1.85-104$.

(1981b). Papel regulador del lenguaje. Infancia y Aprendizaje, Monografia n. ${ }^{\circ} 1$. 115-132.

BowLBY, J. (1969). Attachment and Loss. Vol. I. Londres: Hogarth Press.

BRUNER, J. S. (1964). The Course of cognitive growth. American Psych, 19, 1-15.

(1975). From communication to language, a psychological perspective. Cognitive, 3, 255-287.

(1983a). Investigaciones sobre el desarrollo cognitivo. Madrid: Pablo del Río, Editor.

(1983b). Child talk. Learning to use language. Nueva York: Norton \& Company.

CLARK, E. (1976). Acquisition du langage et représentations sémantiques. Bulletin de Psychologie. N.o especial: La mémoire sémantique, 219-224.

Clarke, A. D., y Clarke, A. M. Recovery from the effects of deprivation. Acta Psychol. 45, pág. 173.

DALE, Ph. (1980a). El desarrollo del lenguaje: un enfoque psicolingüistico. México: Trillas.

(1980b) Is early pragmatic development measurable? J. Child Lang., 7, 1-12.

DORE, J. (1975). Holophrases, speech acts and language universals. J. Child Lang,, 21-40.

(1974). A pragmatic description of early language development, J. Child Lang., 4, 343-350.

HERNÁNDEZ, P. F. (1984). Teorias psicosociolingüísticas y su aplicación a la adquisición del español como lengua materna. Madrid: Siglo XXI.

LOPEZ, S. F. (1981). Los orígenes de la socialización: la vinculación afectiva. Infancia y Aprendizaje, 7-18.

LEONTIEv, A. N. (1969). Sobre la formación de las aptitudes. En VV.AA., El bombre y la cultura, México: Grijalbo.

(1973). Los principios del desarrollo mental y el problema del retraso mental. En VV.AA., Psicología y Pedagogia, Madrid: Akal.

LURIA, A. R. (1973). El papel del lenguaje en la formación de conexiones temporales y la regulación del comportamiento en los niños normales y oligofrénicos. En VV.AA., Psicología y Pedagogía, Madrid: Akal.

(1974). Lenguaje y comportamiento. Madrid: Fundamentos.

(1980). Conciencia y lenguaje. Madrid: Pablo de Río, Editor.

Mardomingo, M. J. (1981). Efectos de la institucionalización sobre la conducta del niño durante los tres primeros años de vida. V Simposio Español de Pediatría Social, Tenerife.

MARTINET. A. (1978). Elementos de lingüizstica general. Madrid: Gredos.

MILLER, I. F. (1981). Assessing language production in children. Experimental procedures. Londres: Univ. Park Press.

OleRON, P. (1977). Tratado de psicología del niño. Vol. 6. Madrid: Morata.

(1981). El niño y la adquisición del lenguaje, Madrid: Morata.

REICH, P. A. (1976). The early acquisition of word meaning. J. Child Lang., 3, 117-123. 
REuCHun, M. (1972). Facteurs socio-économiques du développement cognitif. En Milieu et dévelopment. (Trad. cast. París: P.U.F. Madrid: Pablo del Río, 1979).

RONDAL, J. A. (1982). Lenguaje y educación. Barcelona: Editorial Médica y Técnica.

RichelLE, M. (1975). Ia adquisición del lenguaje. Barcelona: Herder.

Siguan, S., M. (1978). De la comunicación gestual al lenguaje verbal. En VV.AA., La génesis del lenguaje. Madrid: Pablo del Río, Editor.

SNOw, C. (1977). Mothers speech research: from input to interaction. En C. Snow y Ferguson (eds) Talking to children. Londres. Cambridge Univ. Press.

(1979). The role of social interaction in language acquisition. En Collins (ed) Children's language and communication, 195-213. Hillsdale, N. J.: Lawrence Erlbaum.

SPIZ, R. (1981). El primer año de vida del niño. Madrid: F.C.E.

SCHAFFER, H. R. (1977). Early interactive development. En Mother infant interaction, Londres: Academic Press.

(1979). La socialización y el aprendizaje en los primeros años. Ponencia presentada en las $I^{2}$ Jornadas Internacionales de Psicología y Educación, Octubre, Madrid, 1979.

SCHAfFer, H. R., y CrooK, Ch. K. (1981). El papel de la madre en el desarrollo social. Infancia y Aprendizaje, 15, 19-37.

TIZARD, B. (1972). Environmental effects on language development: a study of young children in long-stay residential nurseries. Child Development, 43-339.

TURNER, J. (1980). El niño ante la vida: enfrentamiento, competencia y cognición. Madrid: Morata.

VYGOTSKI L. S. (1973). Pensamiento y lenguaje. Buenos Aires: La Pléyade.

(1973). Aprendizaje y desarrollo intelectual en la edad escolar. En Psicología y Pedagogia. Madrid: Akal.

Wallon, H. (1970). La evolución psicológica del niño. Buenos Aires: Psique.

\section{Extended summary}

Language is acquired and developed in an interactive context. Good acquisition and development need a dialectical and dynamical interaction. In the interaction, the adult not only has to take care of the child, but is also an orientator and organizer of the infant, helping the child to organize his experiences. At the same time, the interactive process is determined by the child's language development. So, as the language is developed, the interactive process also progresses. The interactive patterns are related to the communicative context. So that, if there are different interactive contexts, there are also different models and levels of child language development.

In the present study we try to observe the language acquisition and development in two different contexts. We examine the importance and incidence of the interactive context on language acquisition. For this reason, we used two groups of children (two boys and two girls) studied from two to three years of age. Each of group of children have different background - two of them (Vanesa and Pablo) are living in a institution, and the other two (Celia and Carlos) are living with their parents-. Neither the institutional nor the children in families have organical or neurological problems.

Video was used to record data on the linguistical and interactive context. Language development is studied from three linguistical aspects: sintactical, semantical and pragmatical aspects.

First of all, it is worth pointing out that the different model of the organization of daily life is the most characteristic difference between these two groups of children: The institutional context has a far more inflexible structure than the family context. There is an inflexible time-table in the institution. The institutionalised child's daily life in much more repetitive and monotonous than family life. For this reason, the children who live in a family have more opportunities for changing the communicative context and also gives them more chance of discriminating between the different aspects of reality. Moreover, the chil- 
dren who live in a family do not have apparent social, psychological nor affective problems. On the contraty, the institutionalised children tend to suffer these type of problems. Furthermore, the relationship between adult-infant is far more personal in the family than in the institution.

In the analysis of the results of the study, a big difference between both groups of children is found: language acquisition appears later in institutionalised children. Their development is very slow. They make use of morphological categories at a later stage. Their syntactic structures are simple and inflexible. The lexical property of the institutionalised children is reduced and the contents of their utterances are poor. These children do not use the pragmatical function as much as the children in families and a lot of incorrectness in the pragmatic function was observed.

As already indicated, this linguistical insufficiency does not arise from organical nor neurological problems. It is due, perhaps, to their special situation in the institutional context: the inflexible time-table, for example, of their daily life does not stimulate nor help the child to receive an adequate language development. Moreover, the low level of new experiences has a negative effect on the semantical evolution of the institutionalised children. We can observe this well through the analysis of their lexical property and through the analysis of their social cognitive development. There is indeed a big difference between both groups of children.

Finally in the pragmatic analysis, we find the greatest difference between these groups of children. The institutionalised children present important language deficiencies which were not observed in the analysis of other linguistic aspects. Through the pragmatic analysis, we observed that the institutionalised children do not know how to transform their messages in different interactive contexts correctly. This means that the level of their linguistic comprehension is also very poor. 